\title{
Parrel, vinífera aragonesa de la depresión del Ebro. Adaptación a terroir semiáridos de cultivo.
}

\author{
Ernesto Franco ${ }^{1}$, Alberto Pavon ${ }^{2}$, Yolanda Gogorcena ${ }^{3}$, Ricardo López ${ }^{4}$, Javier Andreu ${ }^{5}$, Jesús J. Usón ${ }^{2}$, y \\ Eva Herrero ${ }^{6}$ \\ ${ }^{1}$ Centro de Transferencia Agroalimentaria - Gobierno Aragón. Unidad de Enología. Avda. de Movera, \\ s/n, 50194, Zaragoza (España). \\ ${ }^{2}$ Centro de Transferencia Agroalimentaria - Gobierno Aragón. Unidad de Tecnología y Mejora de la Vid. \\ Avda. de Movera, s/n, 50194, Zaragoza (España). \\ ${ }^{3}$ Estación Experimental Aula Dei - Consejo Superior de Investigaciones Científicas. Laboratorio de \\ Genómica de frutales y vid. Avda. de Montañana, 1005, 50059, Zaragoza (España). \\ ${ }^{4}$ Laboratorio de Análisis de Aromas y Enología - Instituto Agroalimentario de Aragón. Departamento de \\ Química Analítica de la Facultad de Ciencias de la Universidad de Zaragoza, 50009, Zaragoza (España). \\ ${ }_{5}$ Centro de Transferencia Agroalimentaria - Gobierno Aragón. Unidad de Cultivos Leñosos. Avda. de \\ Movera, s/n, 50194, Zaragoza (España). \\ ${ }^{6}$ Centro de Investigación y Tecnología Agroalimentaria de Aragón. Avda. de Montañana, 930, 50059, \\ Zaragoza (España).
}

\begin{abstract}
Resumen. En el marco del proyecto VALOVITIS (Programa POCTEFA 2014-2020) de identificación y evaluación de variedades minoritarias en el Piedemonte Pirenaico, se está trabajando y sometiendo a estudio la variedad PARREL. Tras los resultados obtenidos en sucesivas campañas de vendimia y vinificación semi-industrial, la variedad Parrel destaca como una de las variedades autóctonas de la región de Aragón con mayor potencial para su posterior desarrollo comercial atendiendo a sus propiedades agronómicas, enológicas a su resistencia a enfermedades como el oídio y a su capacidad de adaptación a condiciones climáticas continentales caracterizadas por elevadas oscilaciones térmicas a lo largo del año.
\end{abstract}

\section{Proyecto VALOVITIS}

El trabajo aquí presentado forma parte del Proyecto VALOVITIS (www.valovitis.eu, Programa POCTEFA 2014-2020), que tiene como objetivo la identificación y evaluación agronómica, tecnológica y aromática de variedades desconocidas de la zona de Piedemonte Pirenaica. Los resultados obtenidos en este proyecto pretenden contribuir a la implantación de variedades desconocidas y proporcionar, a través de la innovación, ventajas competitivas en el mercado.

VALOVITIS trabaja en la localización de variedades de vid locales que poseen perfiles sensoriales diferentes, y que además presenten capacidad de adaptación a las nuevas condiciones climáticas y no muestren vulnerabilidad significativa a plagas y enfermedades.

\section{Banco de germoplasma del Centro de Transferencia Agroalimentaria del Gobierno de Aragón}

Desde 1989 el Centro de Transferencia Agroalimentaria (CTA) del Gobierno de Aragón, en su Banco de Germoplasma, ha implantado, conservado e identificado el material vegetal de vid prospectado. La función principal de este banco es evitar la pérdida de biodiversidad. Para ello se trabaja en la prospección y recuperación de variedades antiguas a punto de desaparecer.

En el marco de VALOVITIS y durante los años 2016 y 2017 se ha profundizado en el estudio de más de 40 accesiones de esta colección, a través de la evaluación agronómica, vinificación y análisis sensorial de los vinos producidos. 


\section{La variedad Parrel}

La PARREL es una de las variedades sometidas a estudio en este trabajo. Se trata de una variedad minoritaria localizada exclusivamente en Aragón y que muestra características agronómicas y enológicas peculiares que merecen un estudio en profundidad de su potencial.

Esta variedad, siendo "desconocida", no está incluida en la relación de variedades de viñedo de vinificación autorizadas en Aragón.

\subsection{La vinífera}

La uva de Parrel es de hollejo tierno y color intenso casi negro.

El origen del material vegetal introducido en el conservatorio del CTA procede de diferentes puntos del valle del Ebro, principalmente en la provincia de Zaragoza, como se muestra en la Figura 1. Los datos climáticos se describen en la Tabla 1.

Tabla 1. Datos climáticos de los lugares de origen del material vegetal de la variedad Parrel evaluados en el proyecto VALOVITIS.

\begin{tabular}{|l|l|c|c|c|c|}
\cline { 2 - 6 } \multicolumn{1}{c|}{} & \multicolumn{1}{|c|}{ Orígen } & $\begin{array}{c}\mathbf{H} \\
(\mathbf{m})\end{array}$ & $\begin{array}{c}\mathbf{P} \\
(\mathbf{m m})\end{array}$ & $\begin{array}{c}\mathbf{E T}_{\mathbf{0}} \\
(\mathbf{m m})\end{array}$ & $\begin{array}{c}\mathbf{T} \\
\left.\mathbf{(}{ }^{\mathbf{}} \mathbf{C}\right)\end{array}$ \\
\hline $\mathbf{1}$ & Ainzón (Z) & 489 & 421 & 1.133 & 14,5 \\
\hline $\mathbf{2}$ & La Almolda (Z) & 355 & 329 & 1.277 & 14,3 \\
\hline $\mathbf{3}$ & Lécera (Z) & 541 & 361 & 1.090 & 13,8 \\
\hline $\mathbf{4}$ & Híjar (TE) & 268 & 335 & 1.208 & 14,6 \\
\hline $\mathbf{5}$ & Daroca (Z) & 894 & 429 & 1.102 & 12,2 \\
\hline $\mathbf{6}$ & Zuera (Z) & 310 & 419 & 1.361 & 14,1 \\
\hline $\mathbf{7}$ & Sástago (Z) & 287 & 339 & 1.324 & 14,9 \\
\hline
\end{tabular}

H: altitud; $\mathrm{P}$ : precipitación anual; $\mathrm{ET}_{0}$ : Potencial de evapotranspiración; T: temperatura anual media; Z: Zaragoza; TE: Teruel. Fuente: Atlas Climático de Aragón.

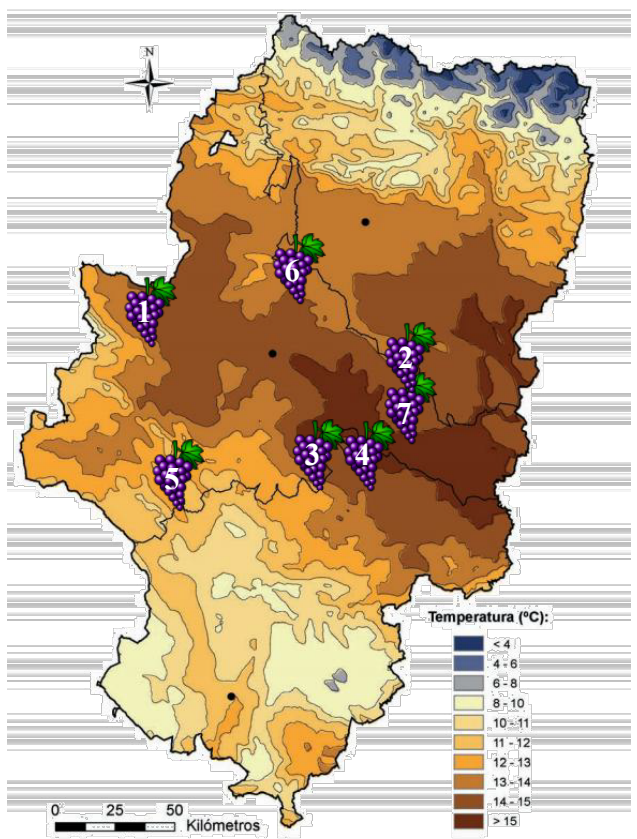

Figura 1. Localización de origen del material vegetal de la variedad Parrel evaluado en el proyecto VALOVITIS. Adaptado del Atlas Climático de Aragón.
La depresión aragonesa del Ebro, es una zona semiárida con precipitaciones anuales no superiores a 400 $\mathrm{mm}$ y temperaturas medias anuales que oscilan entre los 4-6 ${ }^{\circ} \mathrm{C}$ de enero y los $24-26{ }^{\circ} \mathrm{C}$ en julio. La elevada oscilación térmica anual en los lugares en los que ha aparecido esta variedad, induce a pensar que se trata de una variedad con gran capacidad de adaptación a diferentes situaciones climáticas y terroir.

\subsection{Caracterización ampelográfica}

En la Tabla 2 y en la Figura 2 se muestran las características más distintivas de esta variedad.

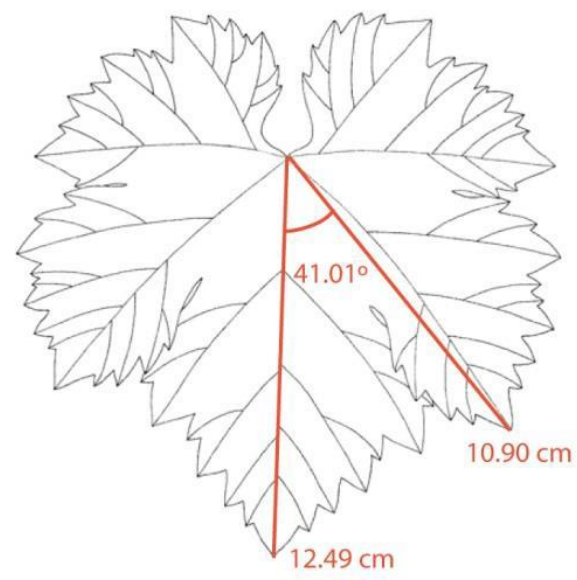

Figura 2. Hoja media reconstruida de Parrel. Análisis ampelométrico. Fuente: Misión Biológica de Galicia-CSIC.

Tabla 2. Descriptores ampelográficos.

\begin{tabular}{|l|l|}
\hline $\begin{array}{l}\text { Pámpano } \\
\text { joven }\end{array}$ & $\begin{array}{l}\text { Distribución de la pigmentación antociánica no } \\
\text { ribeteada y de intensidad media. }\end{array}$ \\
\hline Hoja adulta & $\begin{array}{l}\text { Limbo en forma pentagonal con cinco lóbulos y } \\
\text { dientes rectilíneos. } \\
\text { Densidad de pelos entre los nervios del envés: } \\
\text { media-alta. } \\
\text { Seno peciolar poco abierto y con baja o nula } \\
\text { densidad de pelos. }\end{array}$ \\
\hline Racimo & Mediano y de baja compacidad media. \\
\hline Baya & $\begin{array}{l}\text { Esférica de tamaño medio con una epidermis } \\
\text { azul-negra. }\end{array}$ \\
\hline Pulpa & Sin coloración y con pepitas. \\
\hline
\end{tabular}

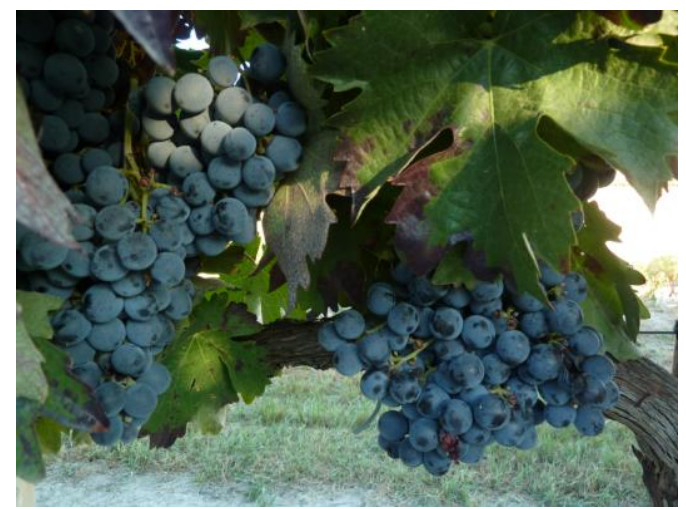

Figura 3. Imagen de los racimos de Parrel. 


\subsection{Caracterización molecular}

Los análisis moleculares realizados en proyectos anteriores con seis marcadores microsatélites, demostraron que se trataba de una variedad no descrita anteriormente y con un perfil molecular no coincidente con los de las bases de datos de décadas pasadas [1].

Tabla 3. Análisis moleculares del material vegetal según su procedencia.

\begin{tabular}{|c|c|c|c|c|c|c|c|}
\hline \multicolumn{2}{|c|}{ VMC4F3-1 } & \multicolumn{2}{|c|}{ VVIN16 } & \multicolumn{2}{c|}{ VVIV37 } & \multicolumn{2}{c|}{ VVIV67 } \\
\hline 179 & 179 & 153 & 159 & 165 & 171 & 364 & 366 \\
\hline
\end{tabular}

\begin{tabular}{|c|c|c|c|c|c|c|c|}
\hline \multicolumn{2}{|c|}{ VVMD27 } & \multicolumn{2}{|c|}{ VVIP31 } & \multicolumn{2}{|c|}{ VVS2 } & \multicolumn{2}{|c|}{ ZAG79 } \\
\hline 179 & 189 & 180 & 196 & 131 & 151 & 243 & 261 \\
\hline
\end{tabular}

Fuente: IMIDRA 2014-15 [2].

La caracterización molecular de varias de las accesiones recuperadas de las zonas de origen, incluidas en la Tabla 1, se llevó a cabo en la Estación Experimental de Aula Dei mediante 8 microsatélites: VMC4F3-1 VVIN16, VVIV37, VVIV67, VVMD27, VVIP31, VVS2 y ZAG79, en el ámbito del proyecto RF2012-00027-C0502 [2]. El perfil molecular obtenido para todas las accesiones fue idéntico independientemente de la zona de origen (Tabla 3). La identificación llevada a cabo en el IMIDRA indica que se trata de la variedad Parrel (GEN 0905) según consta en su base de datos.

En las accesiones que resulten destacadas de este proyecto se deberán llevar a cabo nuevos análisis para completar la caracterización ampelográfica y molecular, con el fin de identificar los biotipos de la variedad que nos permita salvaguardar la variabilidad genética recuperada.

\subsection{Caracterización agronómica}

Durante las campañas de 2016 y 2017, se han realizado vinificaciones de rosado y de tinto con uvas de esta variedad.

Los datos mostrados en las tablas 4 y 5 corresponden a las campañas de 2016 y 2017 vendimiadas en las parcelas del Banco de Germoplasma del Gobierno de Aragón ubicadas en la finca de La Alfranca (Pastriz, Zaragoza). En estas parcelas la conducción se realiza en espaldera y poseen una instalación de riego por goteo. Su ciclo es de secano con algún riego de apoyo si el verano es muy seco.

La variedad Parrel es de ciclo largo y de madurez más tardía que la Garnacha Tinta. Destaca su elevada producción, que se sustenta en el gran número de racimos por cepa y en el tamaño medio de la baya.
Tabla 4. Datos de vendimia de las accesiones utilizadas para la minivinificación de rosados.

\begin{tabular}{|l|c|c|}
\hline Rosado & $\mathbf{2 0 1 6}$ & $\mathbf{2 0 1 7}$ \\
\hline Fecha de vendimia & $4 / 10$ & $16 / 10$ \\
\hline Producción $(\mathrm{kg} / \mathrm{cepa})$ & 8,1 & 5,2 \\
\hline Peso del racimo $(\mathrm{g})$ & 396 & 310 \\
\hline Peso de 100 bayas $(g)$ & 266 & 205 \\
\hline N. de bayas por racimo & 148 & 151 \\
\hline N. de racimos por cepa & 20,5 & 16,9 \\
\hline
\end{tabular}

Tabla 5. Datos de vendimia de las accesiones utilizadas para la minivinificación de tintos.

\begin{tabular}{|l|c|c|}
\hline Tinto & $\mathbf{2 0 1 6}$ & $\mathbf{2 0 1 7}$ \\
\hline Fecha de vendimia & $26 / 9$ & $22 / 9$ \\
\hline Producción por cepa $(\mathrm{kg})$ & 11.7 & 8.2 \\
\hline Peso del racimo $g$ & 586 & 395 \\
\hline Peso de 100 bayas $g$ & 171 & 168 \\
\hline N. de bayas por racimo & 342 & 235 \\
\hline N. de racimos por cepa & 20.0 & 20.8 \\
\hline
\end{tabular}

Se observa que tiene un buen potencial productivo, si las condiciones son favorables, influyendo bastante las condiciones climáticas en el momento del cuajado. Si estas son desfavorables, como en 2016 cuando el cuajado fue irregular, el número de bayas por racimo es menor, lo que provoca una importante minoración productiva de la variedad. El peso de las bayas y el número de racimos por cepa suelen ser valores más constantes que el número de bayas por racimo.

Agronómicamente la variedad Parrel presenta una producción elevada y mantenida en el tiempo, así como una buena resistencia a oídio.

\subsection{Caracterización química - Análisis clásicos}

Durante el proceso de embotellado, un volumen representativo de vino se tomó para analizar, entre otros parámetros, el grado alcohólico adquirido a $20{ }^{\circ} \mathrm{C}$ por absorción de infrarrojos, la acidez total y el $\mathrm{pH}$ a través de una potenciometría automática, el ácido málico por método enzimático (límite de detección: 0,0; límite de cuantificación: 0,1), la intensidad de color por espectroscopia UV-VIS y el índice de polifenoles totales por absorbancia a $280 \mathrm{~nm}$. La inyección en flujo se utilizó para analizar el contenido en azúcares reductores, acidez volátil y el dióxido de azufre total y libre.

Los vinos obtenidos de esta variedad, presentan una analítica adecuada y acorde a las características descritas para vinos rosados y tintos en las Denominaciones de Origen Protegidas (DOP) de Aragón (tabla 6). 
Tabla 6. Análisis moleculares del material vegetal según su procedencia.

\begin{tabular}{|l|c|c|c|c|}
\cline { 2 - 5 } \multicolumn{1}{c|}{} & \multicolumn{2}{c|}{ Rosado } & \multicolumn{2}{c|}{ Tinto } \\
\cline { 2 - 5 } \multicolumn{1}{c|}{} & 2016 & 2017 & 2016 & 2017 \\
\hline $\begin{array}{l}\text { Azucares reductores } \\
(g / l)\end{array}$ & 0,90 & 0,80 & 0,80 & 0,90 \\
\hline $\begin{array}{l}\text { Grado alcohólico } \\
\text { adquirido a 20 }{ }^{\circ} \mathrm{C} \\
(\% \text { v/v) }\end{array}$ & 12,95 & 10,79 & 13,29 & 13,11 \\
\hline Extracto seco total & 21,50 & 23,00 & 24,00 & 26,40 \\
\hline $\begin{array}{l}\text { Acidez total (ác. } \\
\text { Tartárico) }(g / l)\end{array}$ & 5,90 & 5,40 & 5,90 & 5,80 \\
\hline $\begin{array}{l}\text { Acidez volátil (ác. } \\
\text { Acético) }(g / l)\end{array}$ & 0,36 & 0,20 & 0,32 & 0,41 \\
\hline pH & 3,57 & 3,48 & 3,60 & 3,64 \\
\hline Ácido málico (g/l) & 1,40 & 1,50 & 1,30 & 1,70 \\
\hline $\begin{array}{l}\text { Dióxido de azufre total } \\
\text { (mg/l) }\end{array}$ & 17,00 & 44,00 & 33,00 & 102,00 \\
\hline $\begin{array}{l}\text { Dióxido de azufre libre } \\
\text { (mg/l) }\end{array}$ & 5,00 & 5,00 & 5,00 & 7,00 \\
\hline Intensidad de color & 2,50 & 1,55 & 12,43 & 8,26 \\
\hline $\begin{array}{l}\text { Índice de polifenoles } \\
\text { totales }\end{array}$ & 12,20 & 10,40 & 39,60 & 30,00 \\
\hline
\end{tabular}

Análisis: Laboratorio Agroambiental del Gobierno de Aragón.

La graduación alcohólica es moderada, lo que aporta valor añadido a esta variedad si se tiene en cuenta el aumento de grado observado en los vinos aragoneses debido al cambio climático. La acidez y el $\mathrm{pH}$ son normales para los vinos de la región. Destaca la intensidad de color adecuada y suficiente incluso presentando polifenoles totales bajos, circunstancia similar a lo observado en los vinos tintos de Parraleta, variedad minoritaria localizada en la DOP Somontano.

\subsection{Caracterización sensorial}

Los vinos se someten a análisis sensorial, que lo realiza un panel compuesto por 11 jurados expertos de los Centros participantes en el proyecto VALOVITIS (CTA, CITA y LAAE). Se usa para ello una ficha descriptiva donde se valoran descriptores del aroma y del gusto.

En las figuras 4 y 5 , se muestra el perfil sensorial de los vinos rosados y tintos para los dos años de estudio. Es de gran importancia comprobar como los perfiles, que se corresponden con los descriptores valorados en las fichas de cata, son prácticamente iguales con independencia de la cosecha.

En el caso de los rosados, presentan una intensidad media alta, destacando los aromas de carácter fermentativo, mientras que en el gusto destaca la intensidad aromática y la acidez. Los tintos presentan también una intensidad aromática media-alta, destacando por igual el carácter terpénico, especiado y fermentativo. En el gusto destaca la intensidad aromática y la acidez, observando ausencia casi total de los descriptores tánicos.

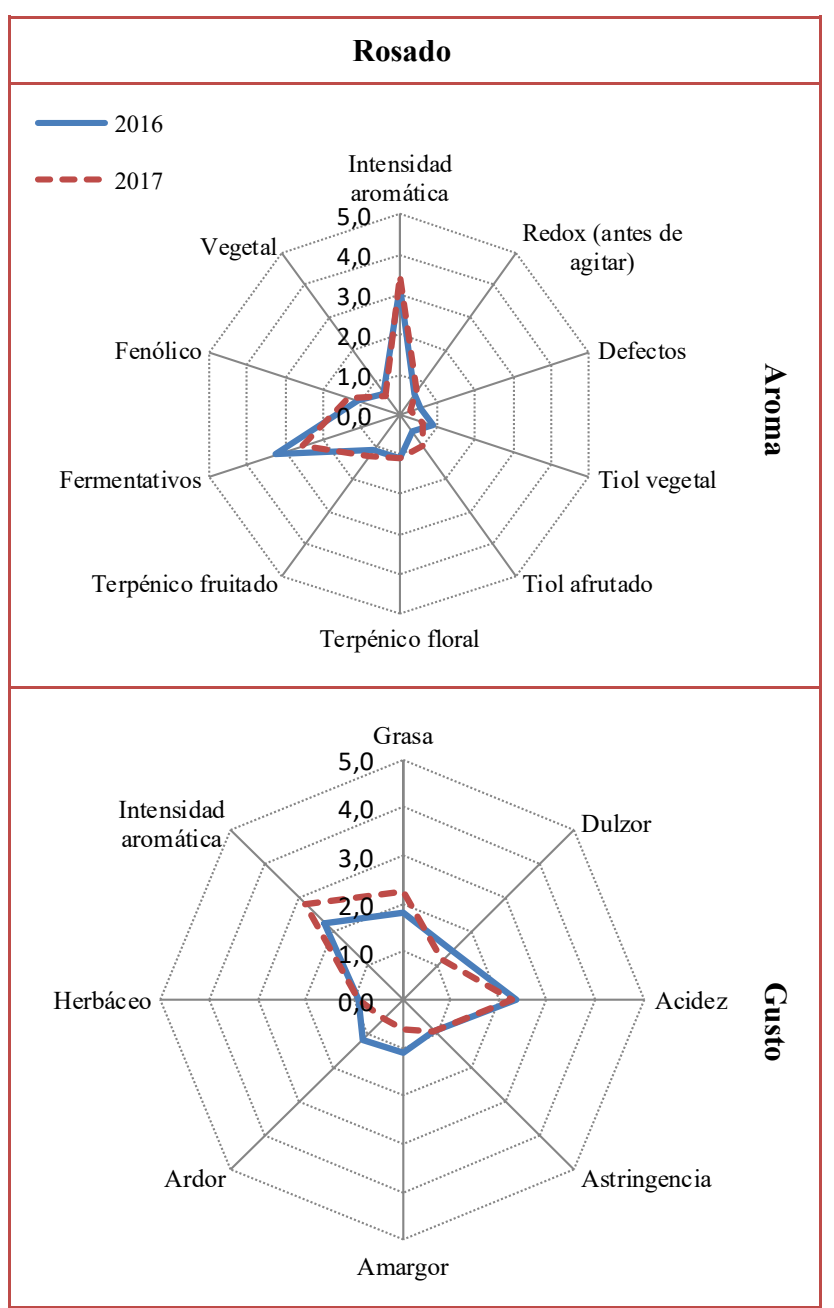

Figura 4. Perfiles aromáticos de la vinificación de rosado obtenidos a partir de la variedad Parrel.

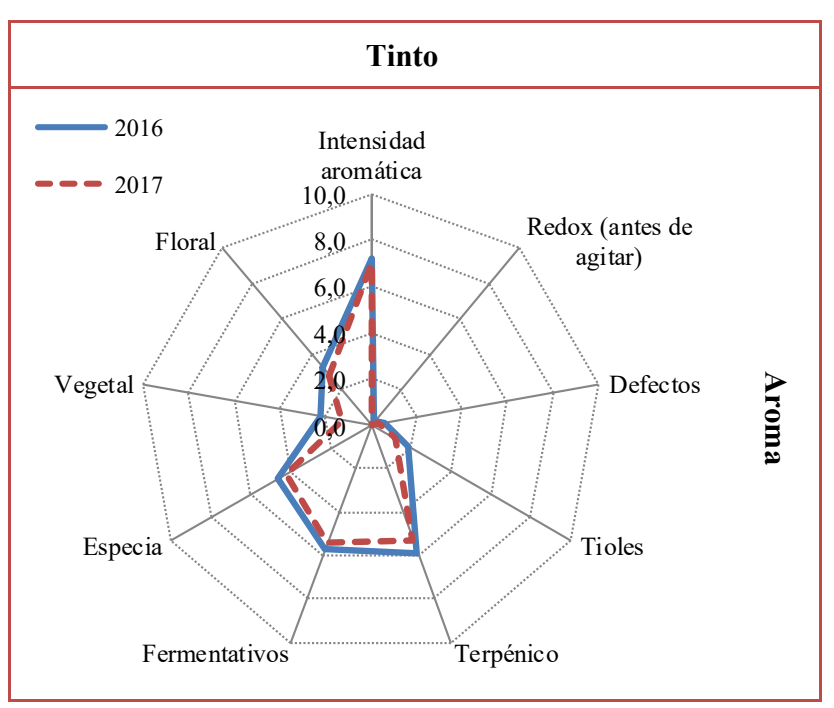




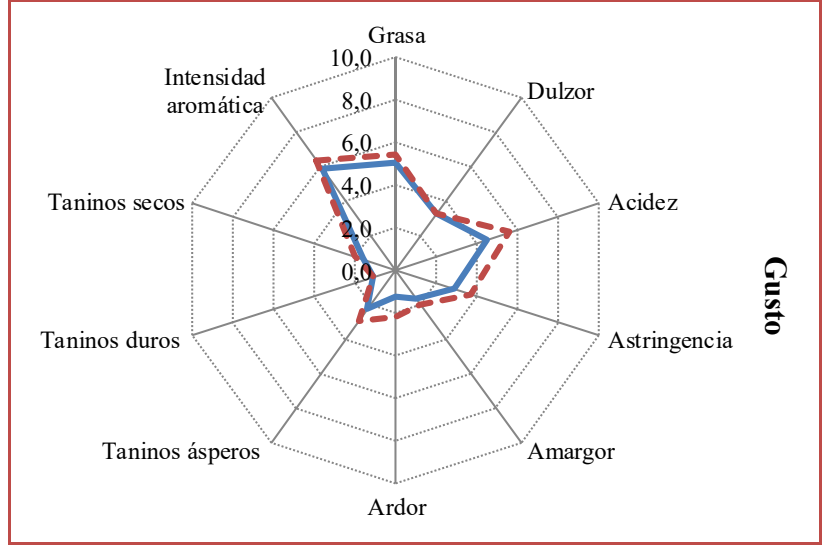

Figura 5. Perfiles aromáticos de la vinificación de tinto obtenidos a partir de la variedad Parrel.

Tanto el grado alcohólico como el contenido fenólico está relacionado con la producción del viñedo. En este caso la elevada producción condiciona que la concentración fenólica sea limitada, incidiendo en que los descriptores de la tanicidad obtengan bajas puntuaciones.

El peso de variedad es muy superior al del año, y mantiene la tipicidad varietal de los vinos, en cuanto a características sensoriales como analíticas.

\section{Conclusiones}

A partir de los resultados de los análisis químicos y sensoriales realizados, se ha observado que los vinos tintos obtenidos presentan un grado alcohólico suficiente $\mathrm{y}$, si bien los componentes fenólicos son bajos, esto no condiciona el potencial de la variedad para producir tanto tintos como rosados de calidad, estos últimos con predominio de aromas terpénicos.

El terroir ha mostrado un efecto menor que la propia caracterización varietal en las producciones obtenidas.

La variedad PARREL, de acuerdo con sus características agronómicas y enológicas, merece poner en valor su potencial a través de estudios posteriores en profundidad sobre su comportamiento agronómico y enológico, en especial en situaciones de producciones más moderadas.

\section{Agradecimientos}

Este proyecto está cofinanciado por el Fondo Europeo de Desarrollo Regional (FEDER).

\section{Referencias}

1. T. Buhner-Zaharieva, S. Moussaoui, M. Lorente, J. Andreu, R. Núñez, J.M. Ortiz, Y. Gogorcena. Am J Enol Vitic, 61, 557-562 (2010).

2. Proyecto RF2012-00027-C5-02. Documentación, caracterización y racionalización del germoplasma de vid prospectado y conservado en España. Creación de una colección nuclear. 